\title{
Transcriptional control of viral gene therapy by cisplatin
}

\author{
James O. Park, ${ }^{1}$ Carlos A. Lopez, ${ }^{1}$ Vinay K. Gupta, ${ }^{1}$ Charles K. Brown, ${ }^{1}$ Helena J. Mauceri, ${ }^{2}$ \\ Thomas E. Darga, ${ }^{2}$ Abdullah Manan, ${ }^{2}$ Samuel Hellman, ${ }^{2}$ Mitchell C. Posner, ${ }^{1}$ \\ Donald W. Kufe, ${ }^{3}$ and Ralph R. Weichselbaum ${ }^{2}$
}

${ }^{1}$ Department of Surgery, and

${ }^{2}$ Department of Radiation and Cellular Oncology, University of Chicago, Chicago, Illinois, USA

${ }^{3}$ Dana-Farber Cancer Institute, Boston, Massachusetts, USA

\begin{abstract}
Ionizing radiation (IR) and radical oxygen intermediates (ROIs) activate the early growth response-1 (Egr1) promoter through specific cis-acting sequences termed CArG elements. Ad.Egr.TNF.11D, a replication-deficient adenoviral vector containing CArG elements cloned upstream of the cDNA for human recombinant TNF- $\alpha$ was used to treat human esophageal adenocarcinoma and rat colon adenocarcinoma cells in culture and as xenografts in athymic nude mice. Cisplatin, a commonly used chemotherapeutic agent, causes tumor cell death by producing DNA damage and generating ROIs. The present studies demonstrate induction of TNF- $\alpha$ production in tumor cells and xenografts treated with the combination of Ad.Egr.TNF.11D and cisplatin. The results show that the Egr1 promoter is induced by cisplatin and that this induction is mediated in part through the CArG elements. These studies also demonstrate an enhanced antitumor response without an increase in toxicity following treatment with Ad.Egr.TNF.11D and cisplatin, compared with either agent alone. Chemo-inducible cancer gene therapy thus provides a means to control transgene expression while enhancing the effectiveness of commonly used chemotherapeutic agents.
\end{abstract}

J. Clin. Invest. 110:403-410 (2002). doi:10.1172/JCI200215548.

\section{Introduction}

Gene therapy for cancer is based on tumor-specific delivery and expression of genes encoding proteins that are directly or indirectly toxic to tumor cells or tumor stro$\mathrm{ma}$ (1). Examples of classes of transgenes used in cancer gene therapy include those that encode prodrugs, cytokines, and immunomodulatory proteins/peptides (2-4). Therapeutic gene delivery systems that include viruses, lipofection, and cell-based and naked DNA have been investigated in preclinical and clinical models (5-7). However, shortcomings in the specificity of tumor targeting and tumor-specific control of gene expression have limited the clinical utility of gene therapy for cancer. Several strategies are currently under investigation for specific delivery of genes to tumors, including genetically altered viruses, specific lipofection formulations, and immunological delivery (8-10).

One potential solution to specific control of gene expression is through the use of tumor- or tissue-selec-

Received for publication March 27, 2002, and accepted in revised form June 13, 2002.

Address correspondence to: Ralph R. Weichselbaum, Department of Radiation and Cellular Oncology, The University of Chicago Hospitals, Center for Advanced Medicine, Room 1329, Mail Code 9006, 5758 S. Maryland Avenue, Chicago, Illinois 60637, USA. Phone: (773) 702-0817; Fax: (773) 834-7233; E-mail: rrw@rover.uchicago.edu.

Conflict of interest: GenVec Inc. provided the vectors used in this study. R.R. Weichselbaum and D.W. Kufe are consultants for GenVec Inc. and hold interest in the company.

Nonstandard abbreviations used: Early growth response- 1 (Egr-1); ionizing radiation (IR); reactive oxygen intermediate (ROI); intratumoral (IT); intraperitoneal (IP); normal saline (NS); luciferase activity (LA). tive promoters to activate transcription of transgenes (11). For example, adenocarcinomas that overexpress MUC-1 preferentially express therapeutic genes that are transcriptionally activated by the MUC-1 promoter (12). Similar strategies have been used for CEA-expressing tumors with the therapeutic constructs containing the CEA promoter (13). Recently, studies have demonstrated physical targeting of gene therapy by IR (14). In this strategy, radio-inducible CArG $\left[\mathrm{CC}(\mathrm{A} / \mathrm{T})_{6} \mathrm{GG}\right]$ DNA elements of the early growth response-1 (Egr1) promoter are ligated upstream of a cDNA encoding human recombinant TNF- $\alpha$ in an adenoviral vector. Using this vector system, TNF- $\alpha$ was preferentially activated in tumors by ionizing radiation (IR) (14). TNF- $\alpha$ is a cytokine secreted by macrophages and other hematopoietic cells that displays antitumor activity in animal studies $(15,16)$. A direct toxic effect on tumor cells, as well as cytotoxic and thrombotic effects on the tumor vasculature, mediates the antitumor effects of TNF- $\alpha$ (17-23). However, systemic toxicities have limited the use of TNF- $\alpha$ in human cancer therapy (24). The combination of TNF- $\alpha$ with chemotherapeutic agents that damage DNA, such as cisplatin and adriamycin, has demonstrated synergistic effects in experimental models $(25,26)$. Recently, isolated limb perfusion with TNF- $\alpha$ and melphalan, a bifunctional alkylating agent, has been reported to be a successful therapeutic strategy for treating limb sarcomas and melanomas (27).

Radiotherapy is a well-developed targeting technology. The efficacy of radiotherapy is limited by tumor radioresistance and the fact that radiotherapy is generally useful against tumors that have not spread beyond the site of origin. Several reports have demonstrated 
additive/synergistic antitumor effects with localized intratumoral expression of TNF- $\alpha$ by a radio-inducible adenoviral vector and IR, without an increase in local or systemic toxicity compared with IR alone $(28,29)$. The TNF- $\alpha /$ IR interaction is cytotoxic to both tumor cells and tumor endothelium, thereby overcoming tumor resistance to either treatment alone (29). Radiotherapy, however, has a limited role in the curative treatment of metastatic disease, and the potential application of radio-inducible gene therapy is limited to local and regional cancer therapy.

Mechanistic studies of Egr-1 induction by IR have demonstrated a role for free radical activation of the CArG elements of the Egr 1 promoter. The role of reactive oxygen intermediates (ROIs) was confirmed by the finding that activation of the Egr1 promoter by $\mathrm{H}_{2} \mathrm{O}_{2}$ is quantitatively and temporally similar to that obtained with IR. Moreover, treatment with $\mathrm{N}$-acetylL-cysteine a free radical scavenger, decreased Egr-1 induction by $\mathrm{IR}$ or $\mathrm{H}_{2} \mathrm{O}_{2}(30)$. These findings have suggested that activation of the Egr1 promoter is mediated by both DNA damage and ROI production. We report herein that cisplatin, a commonly used chemotherapeutic agent that stimulates ROI generation, induces the production of TNF- $\alpha$ in human and rat cancer cells infected with an adenoviral vector encoding the CArG elements of the Egr1 promoter ligated upstream to a cDNA encoding TNF- $\alpha$. Importantly, significant antitumor effects between TNF- $\alpha$ and cisplatin were observed in both experimental tumors. These findings provide support for a new approach that combines cisplatin with the temporal and spatial control of gene therapy.

\section{Methods}

Cells and cell culture. Seg-1, a human esophageal adenocarcinoma cell line (obtained from David Beer, University of Michigan, Ann Arbor, Michigan, USA) and $\mathrm{DHD} / \mathrm{K} 12 / \mathrm{TRb}$ (PROb), a rat colon adenocarcinoma established in syngeneic BD-IX rats by 1,2-dimethylhydrazine induction (obtained from Francois Martin, University of Dijon, France) were maintained in DMEM (Invitrogen Life Technologies, Carlsbad, California, USA) supplemented with FBS (10\% vol/vol) (Intergen Co., Purchase, New York, USA), penicillin (100 IU/ml), and streptomycin $(100 \mu \mathrm{g} / \mathrm{ml})$ (Invitrogen Life Technologies) at $37^{\circ} \mathrm{C}$ with $7.5 \% \mathrm{CO}_{2}$.

Animals. Six-week-old female athymic nude mice (Frederick Cancer Research Institute, Frederick, Maryland, USA) received food and water ad libitum. Experiments were in accordance with the guidelines of the Institutional Animal Care and Use Committee of the University of Chicago.

Viral vectors. Ad.Egr.TNF.11D, a replication-deficient adenovirus constructed to express the human recombinant cytokine TNF- $\alpha$ under the control of the promoter of the Egr1 gene, and control vector Ad.Null.3511.11D, a replication-deficient virus without an effector gene (GenVec Inc., Gaithersburg, Maryland, USA), were stored at $-80^{\circ} \mathrm{C}$ and were diluted to the appropriate concentration in formulation buffer.

In vitro measurement of TNF- $\alpha$ protein. Seg- 1 and $\mathrm{PROb}$ cells were plated at $10^{5}$ cells/well in 12-well plates (Becton, Dickinson and Co., Bedford, Massachusetts, USA), grown overnight, and infected with either Ad.Null.3511.11D or Ad.Egr.TNF.11D at 100 multiplicities of infection in serum-free medium for 2-3 hours. Cells in the IR group were exposed to $5 \mathrm{~Gy}$ in complete medium using a Pantak PCM 1000 x-ray generator (Pantak, East Haven, Connecticut, USA). Cells in the cisplatin group were exposed to $5 \mu \mathrm{M}$ cisplatin in complete medium. Cells and supernatants were harvested at 24,48 , and 72 hours by scraping and human TNF- $\alpha$ production was quantified using a Quantikine ELISA kit (R\&D Systems Inc., Minneapolis, Minnesota, USA) following three freeze-thaw lysis cycles. These experiments were performed in triplicate. Duplicate treatment plates were used to adjust for the cytotoxicity of IR and cisplatin. Cells were harvested using versene (0.02\% EDTA in HBSS) and trypsin-EDTA $(0.25 \%$ trypsin, $1 \mathrm{mM}$ EDTA $\bullet 4 \mathrm{Na}$ ) (Invitrogen Life Technologies), and cells were counted using a hemocytometer with trypan blue $(0.4 \%)$ exclusion (Invitrogen Life Technologies). Protein assays were performed to normalize protein concentration using the Bio-Rad dye reagent (Bio-Rad Laboratories Inc., Hercules, California, USA). Data are expressed as mean \pm SEM of experiments performed in triplicate.

In vitro luciferase reporter assay. The Egr 1 constructs pE425 (596 bp containing all CArG elements and no AP-1 sites) and pE660 (the minimal Egr1 promoter of 115 bp containing no CArG elements) (30) were evaluated following sequence confirmation and insertion of the PCR product into the pGL3 basic firefly luciferase reporter plasmid construct (Promega Corp., Madison, Wisconsin, USA) by enzyme restriction and ligation. JM109 competent cells (Stratagene, La Jolla, California, USA) were transformed with these plasmids, and endotoxin-free maxipreps (QIAGEN Inc., Valencia, California, USA) were prepared. Product confirmation was performed by PCR, sequencing, enzyme restriction, and gel electrophoresis. Seg-1 and PROb cells were plated at $10^{5}$ cells/well in 12-well plates and transfected with the firefly luciferase reporter plasmid constructs pGL3 basic (promoterless, negative control), pGL3 660 (minimal Egr1 promoter), or pGL3 425 (Egr1 promoter containing all CArG elements) - using TransFast transfection reagent (Promega Corp.). All groups were cotransfected with the Renilla luciferase reporter plasmid construct pRL-TK (HSV thymidine kinase promoter) to normalize transfection efficiency. Forty-eight hours later, cells were exposed to IR (20 Gy) or cisplatin $(50 \mu \mathrm{M})$. Cells were harvested 6 hours later, and luciferase activity (LA) was measured using the DualLuciferase reporter assay system (Promega Corp.).

In vivo measurement of TNF- $\alpha$ protein. Seg- 1 or $\mathrm{PROb}$ cells $\left(5 \times 10^{6}\right.$ per $\left.0.1 \mathrm{ml}\right)$ were injected subcutaneously into the right hind limb of nude mice. Tumor-bearing mice were randomized to one of four groups: intratu- 
moral (IT) Ad.Null.3511.11D $\left(2 \times 10^{8}\right.$ particle units [p.u.]/10 $\mu$ l) with intraperitoneal (IP) normal saline (NS) or cisplatin $(8 \mathrm{mg} / \mathrm{kg})$ and IT Ad.Egr.TNF.11D $\left(2 \times 10^{8}\right.$ p.u. $\left./ 10 \mu \mathrm{l}\right)$ with IP NS or cisplatin. IP NS or cisplatin treatments were administered 20 hours after transfection with IT vector, and two consecutive IT and IP injections were given. Animals were euthanized, and xenografts were harvested 48 hours after the second IP injection. Xenografts were snap-frozen in liquid nitrogen and homogenized in RIPA buffer $(150 \mathrm{mM} \mathrm{NaCl}$, $10 \mathrm{mM}$ Tris at $\mathrm{pH} 7.5,5 \mathrm{mM}$ EDTA at $\mathrm{pH}$ 7.5, $100 \mathrm{mM}$ PMSF, $1 \mu \mathrm{g} / \mathrm{ml}$ leupeptin, and $2 \mu \mathrm{g} / \mathrm{ml}$ aprotinin) using a Brinkman Polytron homogenizer (Kinematica AG, Lucerne, Switzerland). After three freeze-thaw lysis cycles, the homogenate was centrifuged at $7800 \mathrm{~g}$ in a Sorvall RC-5C SS34 rotor (Kendro Laboratory Products, Newtown, Connecticut, USA) for 10 minutes at $4^{\circ} \mathrm{C}$. TNF- $\alpha$ levels in the supernatants were measured as described above.

In vivo measurement of TNF- $\alpha$ RNA transcripts using realtime PCR. PROb xenografts were established and treated as in the previous section. Following harvest, tumors were snap-frozen in liquid nitrogen and homogenized in TRIzol reagent (Invitrogen Life Technologies, Carlsbad, California, USA) using a Brinkman Polytron homogenizer. The homogenate was centrifuged at 7800 $g$ in a Sorvall RC5C SS34 rotor for 10 minutes at $4^{\circ} \mathrm{C}$ to remove debris. The samples were chloroform-extracted and total RNA was precipitated with isopropyl alcohol. cDNA was synthesized using SuperScript II reverse transcriptase (Invitrogen Life Technologies), using $5 \mu \mathrm{g}$ total RNA, following the manufacturer's recommendations, but omitting the addition of RNase inhibitor. Quantitative PCR was performed on an ABI Prism 7700 Sequence Detection System (Applied Biosystems, Foster City, California, USA) using SYBR Green PCR reagents (Applied Biosystems) in a $50-\mu 1$ reaction mixture $(5 \mu \mathrm{l} 10 \times$ SYBR Green PCR buffer, $0.5 \mu$ l primers (10 mM), $4 \mu \mathrm{l}$ dNTP mix, $6 \mu \mathrm{lgCl}_{2}(25 \mathrm{mM}), 0.25 \mu \mathrm{l}$ AmpErase, $0.25 \mu \mathrm{l}$ AmpliTaq Gold and $5 \mu \mathrm{l}$ of the $1: 10$ diluted cDNA synthesis reaction product. The final volume was adjusted to $50 \mu \mathrm{l}$ using nuclease-free sterile water. PCR was performed for 40 cycles at $95^{\circ} \mathrm{C}$ for 15 seconds and $60^{\circ} \mathrm{C}$ for 1 minute after initial incubations at $50^{\circ} \mathrm{C}$ for 2 minutes and $95^{\circ} \mathrm{C}$ for 10 minutes. PCR product specificity and purity was evaluated by generating a dissociation curve following the manufacturer's recommendations. Sample CT values were normalized to CT values for mouse GAPDH RNA, all of which were calculated from two PCR reactions performed in triplicate from each of the four tumors. Relative gene induction values were calculated following the manufacturer's recommendations. Note: PROb (rat) xenografts were chosen such that any human TNF- $\alpha$ transcript generated would originate from the Ad.Egr.TNF.11D virus and not from the xenograft tumor cells.

In vivo regrowth studies. Seg- 1 or PROb cells $\left(5 \times 10^{6}\right.$ per $0.1 \mathrm{ml}$ ) were injected subcutaneously into the right hind limb of nude mice. Tumor-bearing mice were assigned to one of four groups: IT Ad.Null.3511.11D $\left(2 \times 10^{8}\right.$ p.u. $/ 10 \mu \mathrm{l})$ with IP NS or cisplatin $(3 \mathrm{mg} / \mathrm{kg})$, and IT Ad.Egr.TNF.11D $\left(2 \times 10^{8}\right.$ p.u./ $\left.10 \mu \mathrm{l}\right)$ with IP NS or cisplatin. Animals were injected IP with NS or cisplatin 20 hours after the IT vector injection. IT and IP injections were given for five consecutive days. Xenografts were measured every 2 days using calipers, and tumor volume was calculated (length $\times$ width $\times$ thickness $) / 2$. Fractional tumor volumes $\left(V / V_{0}\right.$ where $V_{0}=$ volume on day 0 ) were calculated and plotted. Day 0 is the first day of treatment (IT injection vector) and the day that the mice were distributed into treatment groups. Tumor volumes represented on graphs begin on day 0 .

Statistical analysis. Statistical significance was determined using the two-tailed Student $t$ test.

\section{Results}

In vitro induction of TNF- $\alpha$ in buman and rat tumor cells following infection with Ad.Egr.TNF.11D and exposure to cisplatin. Because Egr1 is induced through the CArG elements of its promoter by ROIs and/or DNA damage, we tested the hypothesis that exposure to cisplatin, a DNA-damaging agent that alters cellular redox status (31), will increase TNF- $\alpha$ production by tumor cells infected with an adenoviral vector in which CArG elements are upstream of a TNF- $\alpha$ cDNA (Ad.Egr.TNF.11D). We tested TNF- $\alpha$ production in human esophageal Seg-1 cells and rat colon PROb cells following exposure to $5 \mu \mathrm{M}$ cisplatin. TNF- $\alpha$ concentrations were determined using an ELISA specific to human TNF- $\alpha$. No TNF- $\alpha$ protein was detectable in Seg-1 cell pellets or supernatants from cultures infected with the null vector (Ad.Null.3511.11D) and treated with IR or cisplatin (data not shown). By contrast, significantly increased levels of TNF- $\alpha$ protein were detected at 24,48 , and 72 hours in cultures of Seg- 1 cells infected with the Ad.Egr.TNF.11D vector and exposed to IR (5 Gy) $\left(768.8 \pm 32.6,593.0 \pm 27.6\right.$, and $746.0 \pm 18.5 \mathrm{pg} / 10^{6}$ cells, respectively) compared with cells infected with vector alone $(269.3 \pm 1.9,167.8 \pm 8.4$, and $260.6 \pm 14.9$ $\mathrm{pg} / 10^{6}$ cells, respectively; $\left.P<0.001\right)$. Combined treatment with Ad.Egr.TNF.11D and IR resulted in 2.9-, 3.5- and 2.9-fold increases rounded to the nearest tenth in TNF- $\alpha$ production. A similar induction of TNF- $\alpha$ protein was detected in Seg- 1 cells infected with the Ad.Egr.TNF.11D vector and exposed to 5 $\mu \mathrm{M}$ cisplatin (compared with vector alone) for 24,48 , and 72 hours $(885.3 \pm 28.7,892.6 \pm 21.3,901.7 \pm 21.7$ pg/10 6 cells, respectively; $P<0.001$ ) (Figure 1a). Combined treatment with Ad.Egr.TNF.11D and cisplatin thus resulted in 3.3-, 5.3-, and 3.5-fold increases in TNF- $\alpha$ production.

Comparable experiments were conducted with PROb cell cultures. Again, no TNF- $\alpha$ protein was detectable in PROb cell pellets or supernatants from cultures infected with the null vector (Ad.Null.3511.11D) and treated with IR or cisplatin (data not shown). Significantly increased levels of TNF- $\alpha$ protein were detected in cul- 


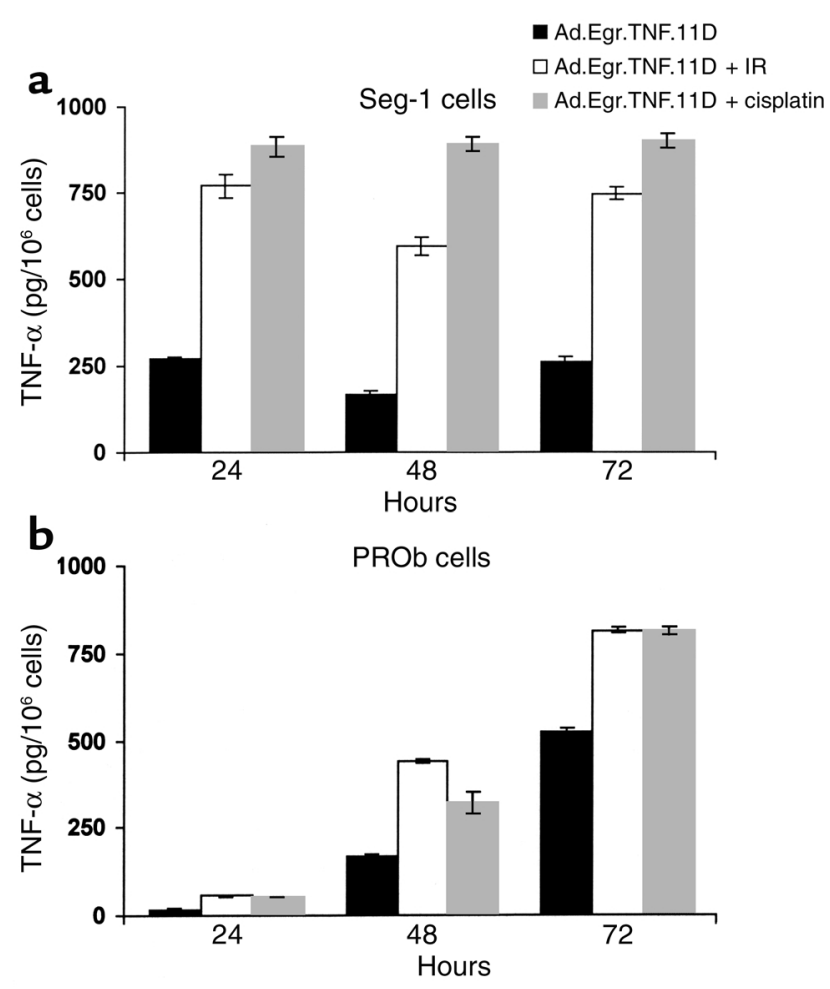

Figure 1

In vitro measurement of TNF- $\alpha$ protein. TNF- $\alpha$ production by Ad.Egr.TNF.11D-infected cells exposed to IR (5 Gy) or cisplatin (5 $\mu \mathrm{M})$ was measured using ELISA. Significant increases in levels of TNF- $\alpha$ protein were detected at 24,48 , and 72 hours following exposure to Ad.Egr.TNF.11D plus IR $(P<0.001)$ and Ad.Egr.TNF.11D plus cisplatin $(P<0.001)$ compared with vector alone in Seg-1 cultures (a) and PROb cultures $(\mathbf{b})$. Data are reported as mean \pm SEM of three independent experiments.

tures of PROb cells infected with the Ad.Egr.TNF.11D vector and exposed to IR (5 Gy) for 24, 48, and 72 hours $\left(55.1 \pm 4.6,440.5 \pm 7.0\right.$, and $812.7 \pm 8.9 \mathrm{pg} / 10^{6}$ cells, respectively) compared with cells infected with vector alone $\left(17.9 \pm 1.7,169.7 \pm 5.2\right.$, and $522.5 \pm 11.3 \mathrm{pg} / 10^{6}$ cells, respectively; $P<0.001)$. Combined treatment with Ad.Egr.TNF.11D and IR resulted in 3.1-, 2.6-, and 1.6fold increases in TNF- $\alpha$ production. A similar induction of TNF- $\alpha$ protein was detected in PROb cells infected with the Ad.Egr.TNF.11D vector and exposed to cisplatin $(5 \mu \mathrm{M})$ (compared with vector alone) for 24,48 , and 72 hours $(52.4 \pm 0.6,318.6 \pm 30.6$, and $812.2 \pm 11.0$ $\mathrm{pg} / 10^{6}$ cells; $P<0.001$ ) (Figure $1 \mathrm{~b}$ ). Combined treatment with Ad.Egr.TNF.11D and cisplatin resulted in 2.9-, 1.9-, and 1.6-fold increases in TNF- $\alpha$ production. These findings from the Seg- 1 and PROb cell lines demonstrate that IR and cisplatin induce TNF- $\alpha$ expression.

CArG elements of the Egr-1 promoter mediate induction of TNF- $\alpha$ by cisplatin. To study whether the CArG elements of the Egr1 promoter are inducible by cisplatin, Egr1 promoter activity was assessed by measuring activation of the luciferase reporter gene in Seg-1 and $\mathrm{PROb}$ cells cotransfected with the firefly luciferase reporter plasmid constructs PGL3 basic (negative con- trol), pGL3 660 (consisting of only the minimal Egr1 promoter, no CArG elements), or pGL3 425 (consisting of all the CArG elements, no AP- 1 sites), and the Renilla luciferase reporter plasmid construct pRL-TK. Minimal LA (expressed as relative luminescence) was detectable in Seg-1 cells transfected with the pGL3 basic plasmid construct (LA $=\sim 0.01-0.02$ ) or with the pGL3 660 plasmid construct $(L A=\sim 0.10-0.18)$. However, Seg-1 cells transfected with the pGL3 425 plasmid construct exhibited a 2.4-fold increase $(P=0.005)$ in relative LA (to 15.07) following exposure to IR (20 Gy) compared with untreated control $(\mathrm{LA}=6.37)$, and a 2.0-fold increase $(P=0.005)$ in LA (to 12.89) following exposure to cisplatin $(50 \mu \mathrm{M})$ compared with untreated control (Figure 2a).

Similar results were obtained with the PROb cell line. Minimal LA was detectable in PROb cells transfected with the pGL3 basic plasmid construct $(\mathrm{LA}=\sim 0.21-0.30)$ or with the pGL3 660 plasmid construct $(\mathrm{LA}=\sim 0.76-1.84)$. PROb cells transfected with the pGL3 425 plasmid construct exhibited a 4.2-fold increase $(P=0.004)$ in LA (to 57.75) following exposure to IR $(20 \mathrm{~Gy})$ compared with untreated control $(\mathrm{LA}=13.69)$, and a 3.6-fold increase $(P=0.01)$ in LA (to 49.40) following exposure to cisplatin $(50 \mu \mathrm{M}) \mathrm{com}$ pared with untreated control (Figure $2 \mathrm{~b}$ ). These data demonstrate that CArG elements of the Egr1 promoter are inducible by cisplatin and mediate the transcriptional activation of the chimeric Egr-TNF- $\alpha$ gene.

Induction of TNF- $\alpha$ in buman and rat tumor xenografts following treatment with Ad.Egr.TNF.11D and cisplatin. We next examined whether TNF- $\alpha$ is induced by cisplatin following infection of human and rodent tumors with the Ad.Egr.TNF.11D vector. Xenografts of Seg-1 or PROb cells growing in the hind limbs of athymic nude mice were injected intratumorally with Ad.Null.3511.11D or Ad.Egr.TNF.11D. Tumor-bearing mice were injected intraperitoneally with either NS or cisplatin $(3 \mathrm{mg} / \mathrm{kg})$. TNF- $\alpha$ concentration in tumor homogenates was quantified using ELISA. No TNF- $\alpha$ protein was detected in Seg-1 tumor homogenates following injection of the Ad.Null.3511.11D vector and systemic treatment with either NS or cisplatin (data not shown). A significant increase (3.5-fold) in IT TNF- $\alpha$ protein was observed following combined treatment with Ad.Egr.TNF.11D and cisplatin $(1,294.0 \pm 438.5 \mathrm{pg} / \mathrm{mg})$ compared with vector treatment alone $(366.5 \pm 52.6 \mathrm{pg} / \mathrm{mg} ; P<0.05)$ (Figure $3 a)$. No TNF- $\alpha$ protein was detected in PROb tumor homogenates following injection of Ad.Null.3511.11D vector and systemic treatment with either NS or cisplatin (data not shown). However, a significant increase $(2.7$-fold) in IT TNF- $\alpha$ protein was observed following combined treatment with Ad.Egr.TNF.11D and cisplatin $(878.6 \pm 61.9 \mathrm{pg} / \mathrm{mg})$ compared with vector treatment alone $(321.4 \pm 27.7 \mathrm{pg} / \mathrm{mg} ; P<0.001)$ (Figure $3 \mathrm{~b})$. These findings demonstrate in vivo induction of TNF- $\alpha$ protein by cisplatin and verify that the TNF- $\alpha$ protein is a product of the Ad.Egr.TNF.11D vector rather than of the tumor tissue. Real-time quantitative PCR analysis 

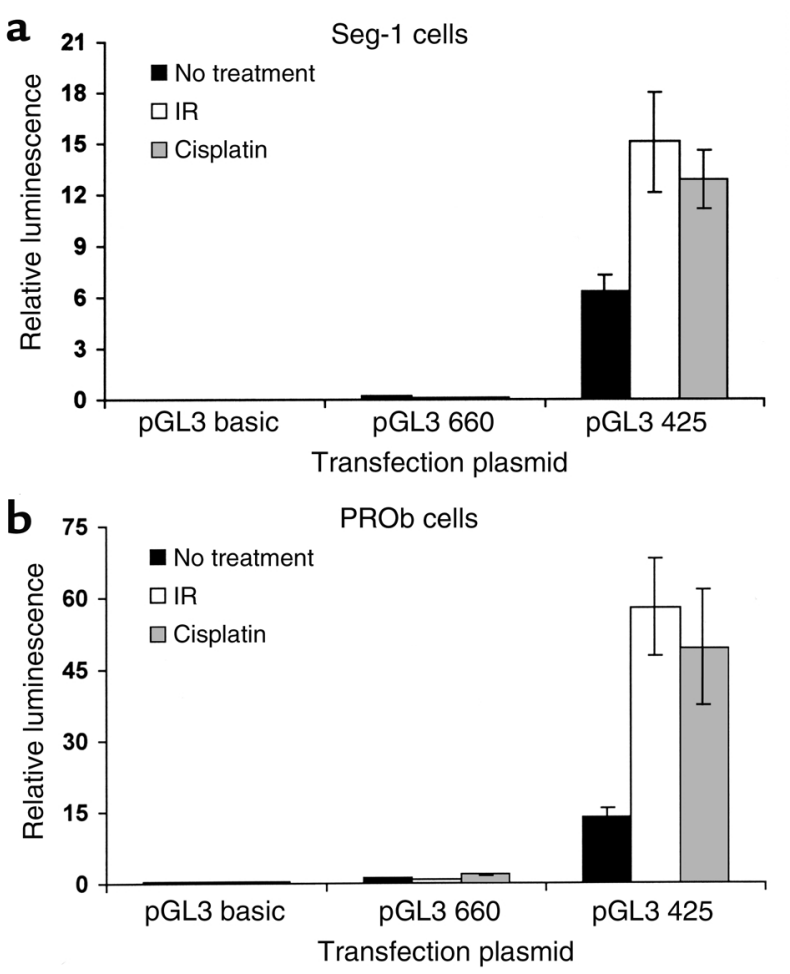

of cDNA synthesized from two cisplatin-treated, Ad.Egr.TNF.11D-infected tumors compared with cDNA from two saline-treated, Ad.Egr.TNF.11D-infected tumors demonstrated an 11-fold induction of human TNF- $\alpha$ transcripts. Sequence verification of the resulting PCR products confirmed human specificity of the PCR primers (data not shown).

Cisplatin-inducible Ad.Egr.TNF.11D enhances treatment of buman and rat xenografts. We examined the potential antitumor effects of chemo-inducible Ad.Egr.TNF.11D and cisplatin in Seg-1 and PROb xenografts. In the Seg-1 studies, mean tumor volume on day 0 (initiation of treatment) was $381.3 \pm 10.8 \mathrm{~mm}^{3}(n=12$ mice per group in each of four treatment groups). Xenografts were injected intratumorally with either Ad.Null.3511.11D or Ad.Egr.TNF.11D. Mice were injected intraperitoneally with either NS or cisplatin. Control tumors (treated with Ad.Null.3511.11D plus NS) doubled in size by day 4 and exhibited a 4.7 -fold increase in mean tumor volume by day 14 . A similar growth pattern was observed in tumors treated with the Ad.Egr.TNF.11D vector and NS (a 2.0-fold increase by day 4 and a 3.8-fold increase in mean volume by day 14). Significant inhibition of tumor growth was observed in the tumors receiving combined treatment with Ad.Egr.TNF.11D and cisplatin compared with tumors treated with the null vector and cisplatin on days $4(P=0.045), 6(P<0.005)$, $8(P<0.002), 10(P<0.001), 12(P<0.004)$, and 14 $(P<0.021)$ after the initiation of treatment (Figure 4a). In the PROb studies, mean tumor volume on day 0 was $244.2 \pm 6.2 \mathrm{~mm}^{3}(n=10$ mice per group in each of four treatment groups). Control tumors (treated with Ad.Null.3511.11D plus NS) grew steadily, dou-

\section{Figure 2}

In vitro reporter assays. Luciferase reporter constructs were used to evaluate induction of the Egr1 promoter by IR or cisplatin. Minimal LA was detectable following transfection with either the PGL3 basic (negative control) or the PGL3 660 plasmid (minimal Egr1 promoter) constructs. (a) In Seg-1 cells transfected with pGL3 425, a 2.4fold increase $(P=0.005)$ in relative LA was observed following exposure to IR (20 Gy), and a 2.0 -fold increase $(P=0.005)$ was seen following exposure to cisplatin $(50 \mu \mathrm{M})$. (b) In PROb cells transfected with pGL3 425, there was a 4.2 -fold increase $(P=0.004)$ in relative LA following exposure to IR (20 Gy), and a 3.6-fold increase $(P=0.01)$ following exposure to cisplatin $(50 \mu \mathrm{M})$. Data are reported as mean \pm SEM.

bling in size by day 4 , exhibiting a 4 .4-fold increase in mean tumor volume by day 14 . A similar growth pattern was observed in tumors treated with the Ad.Egr.TNF.11D vector and NS (1.6-fold increase by day 4 and 3.6-fold increase in mean volume by day 14). Significant tumor regression was observed in the tumors receiving combined treatment with Ad.Egr.TNF.11D and cisplatin compared with tumors treated with the null vector and cisplatin on days $4(P=0.045), 6(P<0.001), 8(P=0.048), 10$ $(P<0.001), 12(P<0.001)$, and $14(P=0.002)$ (Figure $4 \mathrm{~b})$. Taken together, these data support an antitumor interaction between Ad.Egr.TNF.11D and cisplatin in xenografts of human and rodent origin. These findings are consistent with, and supported by, TNF- $\alpha$ induction by cisplatin observed in the in vitro and in vivo experiments. Although toxicity was observed after treatment with cisplatin, no additional toxicity was observed following combined
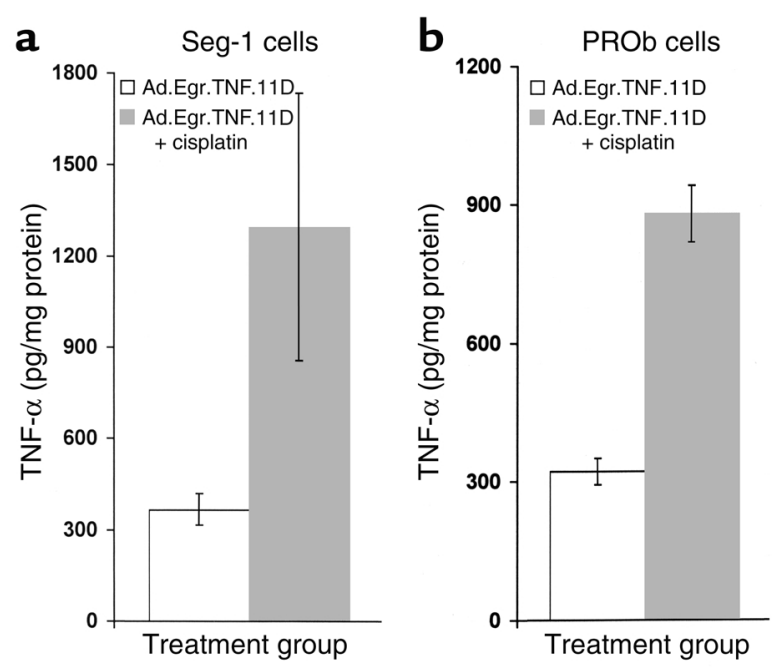

Figure 3

In vivo measurement of TNF- $\alpha$ protein. TNF- $\alpha$ production by Ad.Egr.TNF.11D-injected xenografts was measured by ELISA. A significant increase in ITTNF- $\alpha$ protein concentration was observed following combined treatment with Ad.Egr.TNF.11D and cisplatin compared with treatment with Ad.Egr.TNF.11D vector alone in Seg-1 (a) (3.5-fold increase; $P<0.05)$ and PROb $(\mathbf{b})$ xenografts $(2.7$-fold increase; $P<0.001)$. Data are reported as mean \pm SEM. 

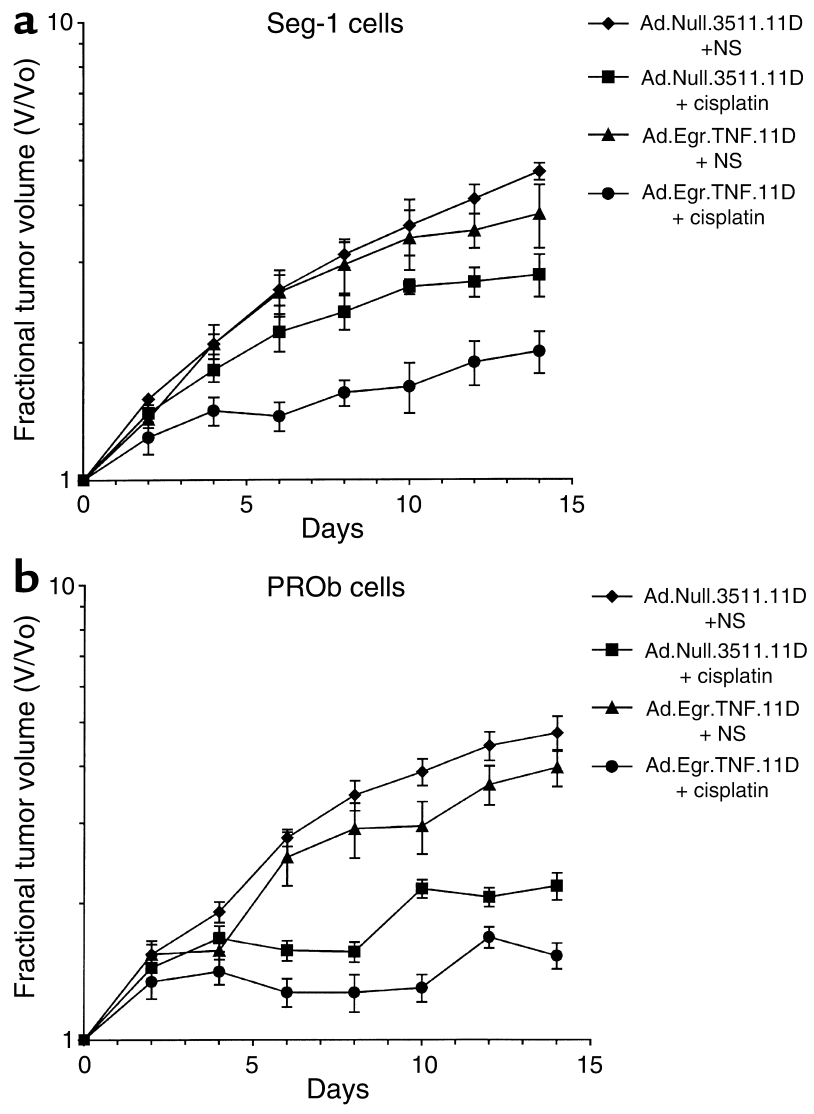

Figure 4

In vivo regrowth studies. The effect of combined treatment with Ad.Egr.TNF.11D and cisplatin was evaluated by measuring the volume of xenografts injected with Ad.Null.3511.11D or Ad.Egr.TNF.11D with or without cisplatin. Day 0 represents the first day of treatment. (a) In Seg-1 xenografts, combined treatment with Ad.Egr.TNF.11D and cisplatin produced significant tumor regression compared with tumors treated with Ad.Null.3511.11D and cisplatin on days $4(P=0.045), 6$ $(P<0.005), 8(P<0.002), 10(P<0.001), 12(P<0.004)$, and 14 $(P<0.021)$. (b) In PROb xenografts, significant tumor regression was observed in the tumors receiving combined treatment with Ad.Egr.TNF.11D and cisplatin compared with tumors treated with Ad.Null.3511.11D and cisplatin on days $4(P=0.045), 6(P<0.001)$, $8(P=0.048), 10(P<0.001), 12(P<0.001)$, and $14(P=0.002)$. Data are reported as mean \pm SEM.

treatment with cisplatin and Ad.Egr.TNF.11D. This antitumor response was achieved despite the fact that infection by IT injection of Ad.Egr.TNF.11D occurred only along the needle track, as was demonstrated using immunohistochemical staining using a $\beta$-gal viral vector (data not shown).

\section{Discussion}

Cisplatin and platinum analogues are currently used in the treatment of head and neck, esophageal, lung, testis, ovarian, and bladder cancers. Additionally, cisplatin is used concurrently with IR as a radiosensitizer. In spite of the relative efficacy of cisplatin, tumor resistance has limited the role of cisplatin in curative cancer chemotherapy (32). Tumor-derived mechanisms of cis- platin resistance include an increase in DNA repair of cisplatin adducts in tumor cells, an increase in glutathione (which inhibits free-radical formation and subsequent DNA damage), and a relative decrease in uptake of cisplatin by resistant cells (33). The combination of cisplatin with other chemotherapeutic agents, especially 5-FU and VP-16, has increased the therapeutic index of both agents in some human tumors (34), but other strategies are needed to increase the efficacy of cisplatin.

TNF- $\alpha$ has been studied as a systemic treatment for cancer in phase I studies, but toxicity has limited the therapeutic index of this cytokine $(24,35)$. Also, combinations of systemic TNF- $\alpha$ and chemotherapy have been investigated in a few clinical trials with limited success (36). Administration of TNF- $\alpha$ in a strategy of isolated limb perfusion with melphalan has demonstrated tumor regression with relatively low systemic toxicity $(37,38)$. We reasoned, therefore, that a high level of IT TNF- $\alpha$ in combination with chemotherapy might provide a greater antitumor response than either agent alone.

The Egr1 gene is induced by a variety of stimuli, including growth factors, IR, and ROIs (39). Transcriptional activation of Egr 1 requires the CArG DNA sequence element in the promoter region of this gene (30). A chimeric genetic construct consisting of the $5^{\prime}$ Egr1 CArG elements ligated to TNF- $\alpha$ cDNA expresses high levels of IT TNF- $\alpha$ following IR exposure of cells transduced with this construct. Tumors transduced with the chimeric Egr-1.TNF construct and treated with IR exhibit increased regression/cure compared with tumors treated with either agent alone, likely due to the IT induction of TNF- $\alpha$ production by IR, and the cytotoxic interaction of TNF- $\alpha$ and IR on the tumor cells and the tumor vasculature $(3,29)$.

The $5^{\prime}$ CArG sequences are known to mediate the induction of Egr1 following exposure to agents that induce intracellular ROIs, as is the case with IR. Therefore, we hypothesized that the use of cisplatin, a commonly used chemotherapeutic agent that alters intracellular radical oxygen formation and damages DNA, might be useful in inducing the TNF- $\alpha$ gene under control of the DNA-damaging and ROI-inducible CArG elements of the Egr1 promoter.

We used an E1/E3/E4-deleted replication-incompetent adenoviral vector containing the chimeric promoter-effector construct Egr-TNF (Ad.Egr.TNF.11D) to deliver the cDNA construct to a human esophageal adenocarcinoma and a rat colon adenocarcinoma. We report that the CArG sequences are activated by cisplatin in vitro when ligated to TNF- $\alpha$ or to the luciferase reporter gene. Additionally, induction of TNF- $\alpha$ by cisplatin was noted in tumor xenografts in vivo. Most importantly, cisplatin induction of TNF- $\alpha$ demonstrates significantly enhanced tumor growth inhibition compared with either agent alone. Moreover, although toxicity was observed following treatment with cisplatin, no additional toxicity was observed with the combination of cisplatin and TNF- $\alpha$. 
There are several potential advantages to using cisplatin as an inducing agent. With a selective tumor-targeting vector, a cisplatin-inducible genetic construct enhances the effects of TNF- $\alpha$, in this case by cisplatin. Cisplatin and TNF- $\alpha$ have different mechanisms of cell killing; therefore, cells resistant to cisplatin may be sensitive to TNF- $\alpha$ and vice versa. Also, necrosis is induced by high IT concentrations of TNF- $\alpha$ through damage to the tumor microvasculature, which may be useful in treatment of TNF- $\alpha-$ and cisplatin-resistant tumors. The cisplatin/Ad.Egr.TNF.11D strategy may thus be an effective therapy for localized tumors that are not effectively treated with radiotherapy or surgery. Also, Ad.Egr.TNF.11D may enhance the local effects of combination chemoradiation therapy.

The use of an inducible promoter in viral gene therapy of cancer has broad potential applicability in oncology practice, as demonstrated in a recently completed phase I trial evaluating the use of Ad.Egr.TNF.11D with radiotherapy. This study included patients with locally advanced/radioresistant melanoma and tumors of the pancreas, head and neck, and breast. Results demonstrate a $60 \%$ complete response-partial response rate and a $30 \%$ stable disease rate, with no added toxicity compared with radiotherapy alone. Several phase II trials in locally advanced head and neck, esophageal, and pancreatic cancer are currently being planned $(\mathrm{H}$. Rasmussen, personal communication). Furthermore, a phase I trial is being designed to evaluate this vector in combination with chemotherapeutic agents (D. Kufe, personal communication).

Control of gene expression is an important issue in gene therapy (40). Our studies demonstrate a potential clinical utility for inducible gene therapy using a genotoxic agent currently used in cancer therapy (cisplatin) and a viral vector containing a promoter with known inducible properties based on DNA damage $(14,30)$. The studies reported herein reinforce the importance and relevance of transcriptional targeting with potentially toxic therapeutic agents under circumstances where tight transcriptional control of gene expression is essential to achieve a high therapeutic index.

For many common human neoplasms, grossly visible tumors are not effectively treated with most standard chemotherapeutic agents. The transcriptional targeting strategy of Egr-TNF and cisplatin may be useful when it is possible to infuse or directly inject macroscopic tumors, even in the presence of micrometastases, since the vector/cisplatin combination is effective against primary tumors and cisplatin is effective against micrometastatic disease. The direct injection of tumors should be improved with the recent advances in radiographic imaging analysis of tumors (e.g., PET scans) combined with computerized tomography (CT) image reconstruction. Additionally, recent developments in the targeting of viral vectors to tumors may provide additional specificity to chemo-inducible gene therapy of metastatic cancer. The use of cisplatin in a strategy of targeting a cisplatin-inducible vector has potentially important implications for improvements in clinical outcome, employing currently used chemotherapies that damage DNA or mediate gene transcription through ROIs.

\section{Acknowledgments}

We thank GenVec Inc. for providing the viral vectors, David Beer for providing the Seg-1 cell line, and Francois Martin for providing the PROb cell line.

1. Hwu, P. 2001. Gene therapy. In Cancer: principles and practice of oncology. V.T. DeVita, S. Hellman, and S.A. Rosenberg, editors. Lippincott, Williams \& Wilkins. Philadelphia, Pennsylvania, USA. 3161-3176.

2. Crystal, R.G., et al. 1997. Phase I study of direct administration of a replication deficient adenovirus vector containing the E. coli cytosine deaminase gene to metastatic colon carcinoma of the liver in association with the oral administration of the pro-drug 5-fluorocytosine. Hum. Gene Ther. 8:985-1001.

3. Weichselbaum, R.R., Kufe, D.W., Advani, S.J., and Roizman, B. 2001. Molecular targeting of gene therapy and radiotherapy. Acta Oncol. 40:735-738.

4. Zwiebel, J.A. 2001. Cancer gene and oncolytic virus therapy. Semin. Oncol. 28:336-343.

5. Bilbao, G., Gomez-Navarro, J., and Curiel, D.T. 1998. Targeted adenoviral vectors for cancer gene therapy. Adv. Exp. Med. Biol. 451:365-374.

6. Dass, C.R., and Burton, M.A. 1999. Lipoplexes and tumours. A review. J. Pharm. Pharmacol. 51:755-770.

7. Yang, J.P., and Huang, L. 1996. Direct gene transfer to mouse melanoma by intratumor injection of free DNA. Gene Ther. 3:542-548.

8. Andreansky, S.S., et al. 1996. The application of genetically engineered herpes simplex viruses to the treatment of experimental brain tumors. Proc. Natl. Acad. Sci. USA. 93:11313-11318.

9. Einfeld, D.A., et al. 2001. Reducing the native tropism of adenovirus vectors requires removal of both CAR and integrin interactions. J. Virol. 75:11284-11291.

10. Rosenberg, S.A. 1996. Development of cancer immunotherapies based on identification of the genes encoding cancer regression antigens. J. Natl. Cancer Inst. 88:1635-1644.

11. Haviv, Y.S., and Curiel, D.T. 2001. Conditional gene targeting for cancer gene therapy. Adv. Drug Deliv. Rev. 53:135-154.

12. Kurihara, T., Brough, D.E., Kovesdi, I., and Kufe, D.W. 2000. Selectivity of a replication-competent adenovirus for human breast carcinoma cells expressing the MUC1 antigen. J. Clin. Invest. 106:763-771.

13. Konishi, F., et al. 1999. Transcriptionally targeted in vivo gene therapy for carcinoembrionic antigen-producing adenocarcinoma. Hiroshima J. Med. Sci. 48:79-89.

14. Hallahan, D.E., et al. 1995. Spatial and temporal control of gene therapy using ionizing radiation. Nat. Med. 1:786-791.

15. Old, L.J. 1985. Tumor necrosis factor (TNF). Science. 230:630-632.

16. Fiers, W. 1991. Tumor necrosis factor. Characterization at the molecular, cellular and in vivo level. FEBS Lett. 285:199-212.

17. Watanabe, N., et al. 1988. Toxic effect of tumor necrosis factor on tumor vasculature in mice. Cancer Res. 48:2179-2183.

18. Tartaglia, L.A., Ayres, T.M., Wong, G.H., and Goeddel, D.V. 1993. A novel domain within the $55 \mathrm{kd}$ TNF receptor signals cell death. Cell. 74:845-853.

19. Robaye, B., Mosselmans, R., Fiers, W., Dumont, J.E., and Galand, P. 1991. Tumor necrosis factor induces apoptosis (programmed cell death) in normal endothelial cells in vitro. Am. J. Pathol. 138:447-453.

20. Havell, E.A., Fiers, W., and North, R.J. 1988. The antitumor function of tumor necrosis factor (TNF). I. Therapeutic action of TNF against an established murine sarcoma is indirect, immunologically dependent, and limited by severe toxicity. J. Exp. Med. 167:1067-1085.

21. Obrador, E., Carretero, J., Pellicer, J.A., and Estrela, J.M. 2001. Possible mechanisms for tumour cell sensitivity to TNF-alpha and potential therapeutic applications. Curr. Pharm. Biotechnol. 2:119-130.

22. Slungaard, A., Vercellotti, G.M., Walker, G., Nelson, R.D., and Jacob, H.S. 1990. Tumor necrosis factor alpha/cachectin stimulates eosinophil oxidant production and toxicity towards human endothelium. J. Exp. Med. 171:2025-2041

23. Mauceri, H.J., et al. 2002. Tumor production of angiostatin is enhanced after exposure to TNF-alpha. Int. J. Cancer. 97:410-415.

24. Spriggs, D.R., et al. 1988. Recombinant human tumor necrosis factor administered as a 24-hour intravenous infusion. A phase I and pharmacologic study. J. Natl. Cancer Inst. 80:1039-1044.

25. Duan, L., et al. 2001. Sensitization of human malignant glioma cell lines to tumor necrosis factor-induced apoptosis by cisplatin. J. Neurooncol. 52:23-36.

26. Bonavida, B., Tsuchitani, T., Zighelboim, J., and Berek, J.S. 1990. 
Synergy is documented in vitro with low-dose recombinant tumor necrosis factor, cisplatin, and doxorubicin in ovarian cancer cells. Gynecol. Oncol. 38:333-339.

27. Thom, A.K., et al. 1995. Cytokine levels and systemic toxicity in patients undergoing isolated limb perfusion with high-dose tumor necrosis factor, interferon gamma, and melphalan. J. Clin. Oncol. 13:264-273.

28. Chung, T.D., et al. 1998. Tumor necrosis factor-alpha-based gene therapy enhances radiation cytotoxicity in human prostate cancer. Cancer Gene Ther. 5:344-349.

29. Staba, M.-J., Mauceri, H.J., Kufe, D.W., Hallahan, D.E., and Weichselbaum, R.R. 1998. Adenoviral TNF-alpha gene therapy and radiation damage tumor vasculature in a human malignant glioma xenograft. Gene Ther. 5:293-300.

30. Datta, R., et al. 1993. Reactive oxygen intermediates target CC(A/T)6GG sequences to mediate activation of the early growth response 1 transcription factor gene by ionizing radiation. Proc. Natl. Acad. Sci. USA. 90:2419-2422.

31. Davis, W., Jr., Ronai, Z., and Tew, K.D. 2001. Cellular thiols and reactive oxygen species in drug-induced apoptosis. J. Pharmacol. Exp. Ther. 296:1-6

32. Johnson, S.W., and Stevenson, J.P. 2001. Cisplatin and its analogues. In Cancer: principles and practice of oncology. V.T. DeVita, S. Hellman, and S.A Rosenberg, editors. Lippincott, Williams \& Wilkins. Philadelphia, Pennsylvania, USA. 376-388.
33. Kartalou, M., and Essigmann, J.M. 2001. Mechanisms of resistance to cisplatin. Mutat. Res. 478:23-43.

34. Kucuk, O., Shevrin, D.H., Pandya, K.J., and Bonomi, P.D. 2000. Phase II trial of cisplatin, etoposide, and 5-fluorouracil in advanced non-smallcell lung cancer: an Eastern Cooperative Oncology Group Study (PB586). Am. J. Clin. Oncol. 23:371-375.

35. Demetri, G.D., et al. 1989. A phase I trial of recombinant human tumor necrosis factor and interferon-gamma: effects of combination cytokine administration in vivo. J. Clin. Oncol. 7:1545-1553.

36. Nakamoto, T., Inagawa, H., Takagi, K., and Soma, G. 2000. A new method of antitumor therapy with a high dose of TNF perfusion for unresectable liver tumors. Anticancer Res. 20:4087-4096.

37. Libutti, S.K., Barlett, D.L., Fraker, D.L., and Alexander, H.R. 2000. Technique and results of hyperthermic isolated hepatic perfusion with tumor necrosis factor and melphalan for the treatment of unresectable hepatic malignancies. J. Am. Coll. Surg. 191:519-530.

38. Lans, T.E., et al. 2001. Role of tumor necrosis factor on toxicity and cytokine production after isolated hepatic perfusion. Clin. Cancer Res. 7:784-790.

39. Weichselbaum, R.R., Hallahan, D., Fuks, Z., and Kufe, D. 1994. Radiation induction of immediate early genes: effectors of the radiation-stress response. Int. J. Radiat. Oncol. Biol. Phys. 30:229-234.

40. Crystal, R.G. 1995. Transfer of genes to humans: early lessons and obstacles to success. Science. 270:404-410. 